\title{
Constrains on the extragalactic origin of IceCube's neutrinos using HAWC
}

\author{
Ignacio Taboada \\ Georgia Institute of Technology \\ E-mail: itaboada@gatech.edu
}

\section{Chun Fai Tung}

Georgia Institute of Technology

E-mail: christung616@gmail.com

\section{Joshua Wood*}

University of Wisconsin at Madison

E-mail: jwoodeumdgrb.umd. edu

\section{for the HAWC Collaboration ${ }^{\dagger}$}

\begin{abstract}
IceCube has discovered an unresolved and isotropic flux of neutrinos between $10 \mathrm{TeV}$ and $8 \mathrm{PeV}$. Extragalactic origin for this flux is usually assumed, as well as a correlation with the sources of cosmic rays. To date, no clear association with a class of objects has been made. HAWC is a very-high-energy (VHE, or $\gtrsim 100 \mathrm{GeV}$ ) gamma ray observatory in operation in central Mexico. HAWC has studied $2 / 3$ of the entire sky $(\sim 8 s r)$. We use this survey to search for optically thin sources of cosmic rays and neutrinos, responsible for IceCube's observations. We have written a simulation of the cosmological properties of neutrino sources, FIRESONG, to show that under certain conditions, HAWC should be observing multiple sources. However, HAWC has only detected 2 extragalactic objects in 760 days of livetime: Mrk 421 and Mrk 501. This deficiency of detected sources in the extragalactic sky can be used to constrain the properties of neutrino sources, such as their density in the local universe. The case HAWC restricts the most is for no evolution in the density of sources, in which at least 8 objects should have been detected for the cases not excluded already by IceCube.
\end{abstract}

35th International Cosmic Ray Conference - ICRC2017

10-20 July, 2017

Bexco, Busan, Korea

\footnotetext{
* Speaker.

${ }^{\dagger}$ Complete list of authors at http://www.hawc-observatory.org/collaboration/icrc2017.php
} 


\section{Introduction}

IceCube has observed astrophysical neutrinos in the $10 \mathrm{TeV}-8 \mathrm{PeV}$ energy range $[1,3]$. Though neutrino directions are known to $\sim 1^{\circ} 1$, IceCube has not resolved neutrino sources. In IceCube's lingo, a point source has not been found. [5]. There is not clear association of a class of objects with astrophysical neutrinos. Multiple candidates classes have been proposed including Blazars, GRBs, Starburst galaxies, etc. Isotropy is usually interpreted as indication of extragalactic origin, however this is unproven. Finding the sources of the astrophysical neutrinos is one of the outstanding questions in particle astrophysics.

If neutrino sources are cosmic ray sources and are optically thin, then neutrinos are due to $\pi^{ \pm}$ decay and gamma rays are produced via $\pi^{0}$ decay. A common strategy to try identify the sources is to search for an electromagnetic counterpart by pointing an instrument, from radio to very-highenergy (VHE) gamma rays, in the direction of individual neutrinos reported by IceCube. In these proceedings we pursue a different approach, we use $2 / 3$ of the entire sky that has been surveyed by the HAWC gamma ray observatory.

We will show that for a wide range of astrophysical and cosmological options, if neutrino sources are extragalactic, HAWC should detect multiple nearby sources. Using data collected over 760 days, HAWC has observed 2 extragalactic sources: Mrk 421 and Mrk 501 [6]. The relatively low number of observed sources can be explained if: a) Sources are transient - currently we have only examined time integrated HAWC data. b) The sources of neutrinos are optically opaque. c) The neutrino spectrum has a break at energies lower than what has been observed by IceCube. d) the local density of neutrino sources is higher than $10^{-6} \mathrm{Mpc}^{-3}$ and sources evolve proportional to star formation history (SFH).

\section{HAWC}

HAWC is a gamma-ray observatory in operation in central Mexico at an elevation of 4,100 $\mathrm{m}$ asl. HAWC operates with $>95 \%$ duty cycle with a field of view of 2 sr. With Earth rotation, HAWC observes $2 / 3$ of the sky every day. HAWC is sensitive from a few hundred GeV to over $100 \mathrm{TeV}$ gamma rays. HAWC was inaugurated on March 20, 2015, but its modular construction allowed operation before that time. Results presented here correspond to 760 days of effective livetime collected between November 2014 and February 2017. Using a 507 day effective livetime dataset, HAWC has reported the observation of 39 sources [6].

HAWC uses the water Cherenkov technique, in which VHE photons are detected by measuring Cherenkov light from ground level secondary particles in an extensive air shower. HAWC consists of 300 steel tanks each containing a light-tight bladder of $7.3 \mathrm{~m}$ diameter and $4.5 \mathrm{~m}$ in height. Each tank holds $\sim 200,000$ liters of filtered water. Charged particles traveling faster than the speed of light in water produced Cherenkov radiation. A 10" photomultiplier tube (PMT) and three 8" are placed at the bottom of each tank to capture the Cherenkov light. Relative Cherenkov arrival times at each PMT determine the direction of the shower plane, and hence the primary gamma ray. Background is mostly cosmic ray air showers. The lateral distribution function for cosmic rays and gamma rays air showers differ. The former includes muons, that often land far away from the

\footnotetext{
${ }^{1}$ For the track channel, mostly due to CC interactions of $v_{\mu}$. Cascades, sensitive to all flavors, have poorer pointing.
} 
shower core; the latter is smoother as a function of distance to the shower core. These two facts are used by HAWC data analysis techniques to separate signal from background.

In these proceedings we use HAWC detailed background measured with direct integration [8] and the current best existing model of HAWC's instrument response (aka HAWC pass 4.1), including the point spread function. HAWC analysis techniques for pass 4.1 are detailed in Ref. [7].

\section{The neutrino-gamma ray connection}

By correlating neutrino directions and times, IceCube has ruled out multiple source classes as the source of astrophysical neutrinos, including Gamma ray bursts [4], Blazars [13], and nearby starburst galaxies [5]. If neutrino sources are cosmic ray sources, then the charged to neutral pion ratios allows the calculation of the expected gamma ray flux [11], with $\mathrm{p}-\gamma$ neutrino production yielding $\sim 2 \times$ more neutrinos than $\mathrm{p}-\mathrm{p}$ interactions.

The astrophysical neutrino spectrum is usually fitted by IceCube using a power law. There may be a tension between the spectral indices measured with tracks, $\sim-2.1$ [3], and cascades $\sim-2.7$ [10]. The discrepancy can be explained with two neutrino populations with different spectral indices and noting that tracks are only sensitive above $\sim 100 \mathrm{TeV}$, while cascades are sensitive to lower energy. We do not address this issue in these proceedings. Instead we assume a soft spectral index, since this is the best description of IceCube's lower energy range, tens of TeV, in which HAWC also has sensitivity. We adopt as reference the spectrum obtained in a joint study of multiple IceCube measurements [2] with an index of -2.5.

VHE gamma rays propagating over cosmological distances are attenuated via the interaction with extragalactic background light (EBL). HAWC's detectable range is $z \lesssim 0.1$ due to EBL attenuation. At energies higher than $\sim 300 \mathrm{TeV}$, attenuation is due to interaction with the cosmic microwave background and observable distances are of galactic scale only. Also, HAWC's sensitivity beyond $100 \mathrm{TeV}$ (with pass 4.1) is not well understood. Hence in these proceedings we cutoff gamma ray spectra at $100 \mathrm{TeV}$.

\section{Euclidean cosmology of neutrino sources}

The astrophysical neutrino flux is proportional to the characteristic luminosity of the sources in neutrinos $L_{v}$ and the density of sources $\rho$. The density, may evolve with redshift, so we denote $\rho_{0}$ as the local density. It follows that the local power density, $\rho_{0} L_{v}$ is a constant proportional to the flux measured by IceCube. Using an Euclidean approximation, it can be shown [16] that:

$$
\rho_{0} L_{v} \sim \frac{4.4 \times 10^{43}}{\xi} \frac{\mathrm{erg}}{\mathrm{Mpc}^{3} \cdot \mathrm{yr}}
$$

where $\xi$ is a factor in the range 0.6-8 that measures the evolution of $\rho$. Using the simulation described in $\S 5$, a similar relationship at eqn.4.1 is obtained. For evolution proportional to SFH, $\xi \sim 2.6$ and with no evolution, $\xi \sim 0.6$. SFH history evolution and no evolution are representative of a very large number of classes of potential sources of neutrinos such as starburst galaxies and blazars respectively. 


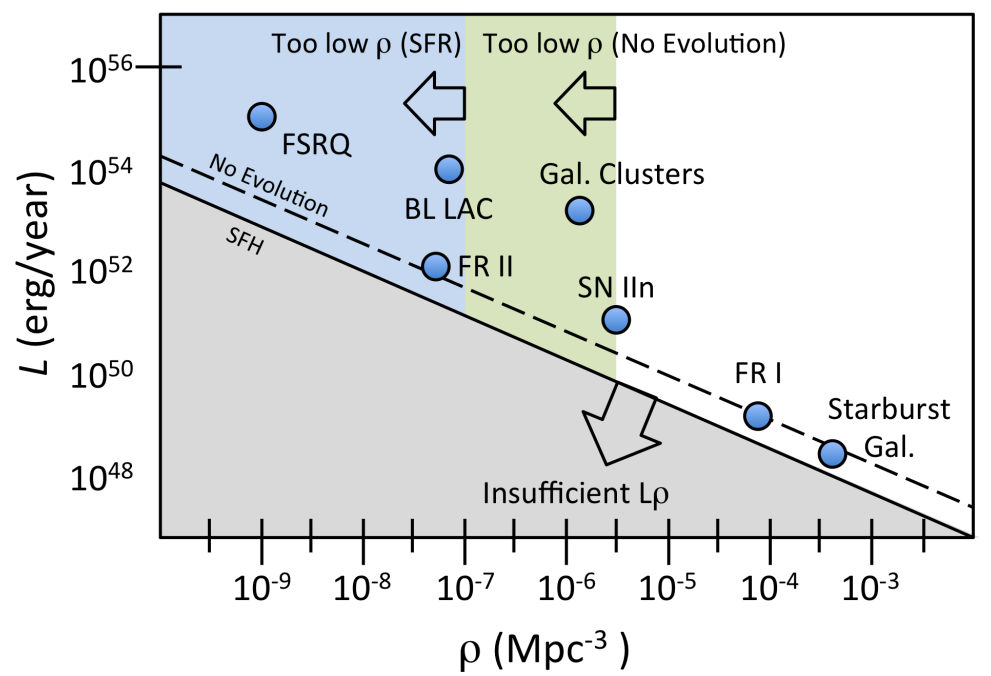

Figure 1: Constrain on local power density $\left(\rho_{0} L_{v}\right)$ are shown as a diagonal lines assuming $L_{v} / L_{\gamma}=1$ and SFH evolution for source density (solid) or no evolution (dashed). Also shown are lower bounds on local density of steady sources set by IceCube's lack of observation of resolved sources for SFH and no evolution cases.

The argument above assumes that sources are steady. A very similar conclusion is reached for transient sources but with the power density substituted by $\dot{\rho}_{0} E_{0}$, where $\dot{\rho}$ is the density rate of bursts and $E_{0}$ is the characteristic energy released by a single burst. In these proceedings we only study the steady case.

A separate constrain can be derived from the lack of detection of point sources by IceCube. The brightest (and thus nearby) sources must have a luminosity low enough to satisfy the lack of resolved sources. Via the constrain on the power density, this lack of resolved sources implies a lower bound on neutrino sources, $\rho \gtrsim 10^{-7} \mathrm{Mpc}^{-3}(3 / \xi)^{3}$. Figure 1 shows a summary of constrains on density and power density as set by IceCube for steady sources. The reader should note that the characteristic electromagnetic luminosity $L_{\gamma}$ may be known, but the ratio $L_{v} / L_{\gamma}$ is not. Source opacity, may result in $L_{v} / L_{\gamma}>1$ while non-hadronic processes would imply the opposite.

\section{FIRESONG: a simulation of neutrino source cosmology}

We have written a simulation of neutrino sources, FIRESONG, that replicates $\S 4$, but using $\Lambda \mathrm{CDM}$ cosmology. The total flux of all neutrino sources simulated is made to match IceCube's observations, e.g. Ref. [2]. FIRESONG includes many choices of source evolution, such as SFH as determined by Hopkins and Beacom [14] and Candels and Clash for evolution following core collapse supernovae[18]. FIRESONG also allows for choice of luminosity functions, such as power laws, lognormal distributions, standard candles, etc. The code is very modular and easily allows coding of additional density evolution and luminosity functions, including evolution of the luminosity function. Though in these proceedings we only deal with steady sources, FIRESONG can also simulate transient sources. FIRESONG can be used in two modalities. In the mode used in the current proceedings the local density of sources is a free parameter. Once this has been set, the output is the list of all neutrino sources in the universe, described by their neutrino flux 


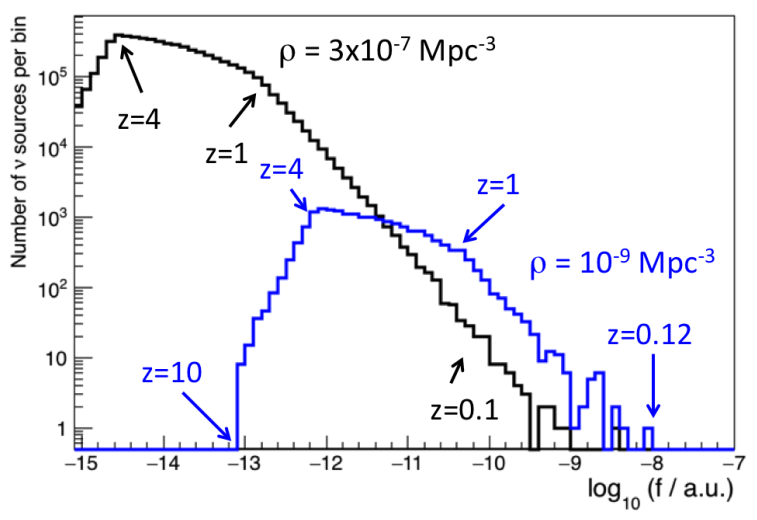

Figure 2: Sample FIRESONG output for two choices of local density of neutrino sources. These two simulation runs assumed SFH evolution and standard candle luminosity function.

at Earth and redshift. A separate mode, not used here, produces individual neutrino events, also described by neutrino flux and redshift. This latter mode can be used by observatories that point in the direction of individual neutrinos to try to find an electromagnetic counterpart. FIRESONG code and documentation can be downloaded from [19].

A sample output of FIRESONG can be seen in fig. 2. The panels show the number of sources for a given neutrino flux at Earth for two choices of local density. The simulation was performed assuming SFH evolution [14] and with standard candle sources. This choice of luminosity function means a direct mapping between flux at Earth and redshift. Various redshifts are indicated in the figures. The highest redshift considered is 10 . At these redshift, evolution of density and luminosity is very uncertain, however, the bulk of the contribution to the neutrino flux observed at Earth is for $z \lesssim 2$.

Fig. 2 shows that, as expected, higher local density means more sources in the Universe. But also as described in section $\S 4$, higher density results in lower luminosity (flux) sources. For a density of $3 \times 10^{-7} \mathrm{Mpc}^{-3}$ multiple sources in the Universe are at a redshift of 0.1 or lower, potentially detectable by HAWC. But for density $10^{-9} \mathrm{Mpc}^{-3}$ the nearest source in this simulation run had a redshift of 0.12 - possibly at the edge of what HAWC can detect.

\section{All sky sensitivity of HAWC to neutrino sources}

To convert neutrino fluxes produced by FIRESONG we assume p-p neutrino production because this is the more pessimistic scenario for gamma rays. We also apply EBL attenuation following Gilmore et al. [12]. Source location in the sky, $\delta$ and RA, are randomize assuming isotropy. Each FIRESONG run results in a list of nearby sources described by and EBL attenuated gamma ray spectrum and a location in the sky.

We use HAWC's software to inject the sources from each FIRESONG run into a background template map measured by HAWC using direct integration. Then the signal strength (in significance) is determined. The determination of the significance of a source uses HAWC's knowledge of the point spread function for gamma rays in terms of, e.g. declination and gamma ray energy ${ }^{2}$.

\footnotetext{
${ }^{2}$ Event size actually, which is correlated with primary energy.
} 

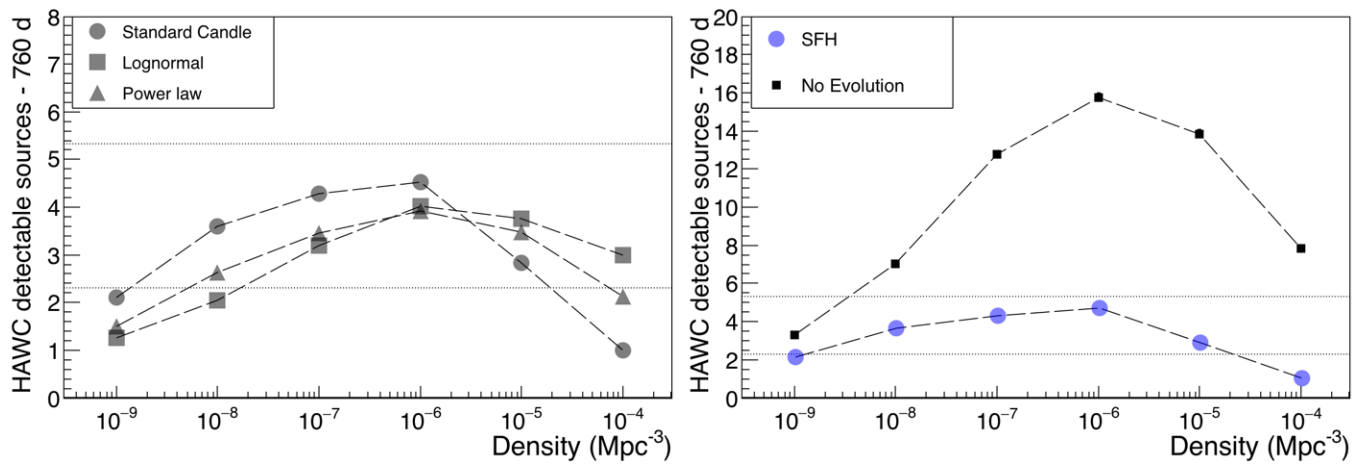

Figure 3: Number of sources detectable by HAWC in 760 days of operation. Left pannel corresponds source density that evolves following SFH [14]. Various choices of luminosity functions are shown. The right panel compares standard candle sources with evolution as SFH and no evolution.

In HAWC, to achieve a post-trial significance over the entire sky above 5 sigma is approximately equivalent to a pre-trial significance of 7.5 sigma. We consider a FIRESONG simulated source detectable if it exceeds 7.5 sigma pre-trial using HAWC's software.

Figure 2 provides guidance into the range of local densities HAWC can explore. At very low densities, even the nearest sources are too far to be detectable by HAWC due to EBL attenuation. Using $\S 4$ it can be shown that the flux of the nearest sources depends on $\rho^{-1 / 3}$. As the density increases, the nearest sources have a redshift that is more favorable for HAWC but the flux only decreases slowly. When the density is very high, there's little or no EBL attenuation at all (i.e. when there are multiple nearby sources at a few tens of Mpc). Beyond this density, the number of HAWC detectable sources diminishes as sources become weaker and weaker with increasing density. In summary, we expect HAWC to be most sensitive to intermediate values, $\rho 10^{-7}-10^{-5} \mathrm{Mpc}^{-3}$ of local density of neutrino sources.

Figure 3 shows the number of FIRESONG predicted detectable sources by HAWC in 760 days of operation. The left panel shows density evolving as SFH for various luminosity functions: Standard candles, a power law luminosity function $d N / d L \propto L^{-2}$ over 3 orders of magnitude in the range of luminosity and a lognormal function with a width of proportional to the average luminosty, $L_{v}$. The various choices of luminosity function only result in a factor of $\sim 2$ in the number of detectable sources, expect at the highest density considered. However, a factor of $\sim 3-5$ resuls from a change from SFH evolution to no evolution (right panel fig. 3). The resason why the no evolution is more favorable for HAWC is that the number of nearby sources does not change much. Within redshift 0.1 is $\sim 23$ for SFH evolution and $\sim 17$ for no evolution. But for no evolution, the luminosity is $\sim 4$ times larger than for SHF, as expected from eqn. 4.1. Figure 4 shows standard candles for 5 years of HAWC operation with SFH and no evolution.

As a guideline of whether these calculations are inconsistent or not with HAWC observations, figures 3 and 4 show horizontal dashed lines at 2.3 (5.3) detectable sources. These are the numbers at which a given prediction is inconsistent at $90 \%$ C.L. with an observation of zero (two) sources. 


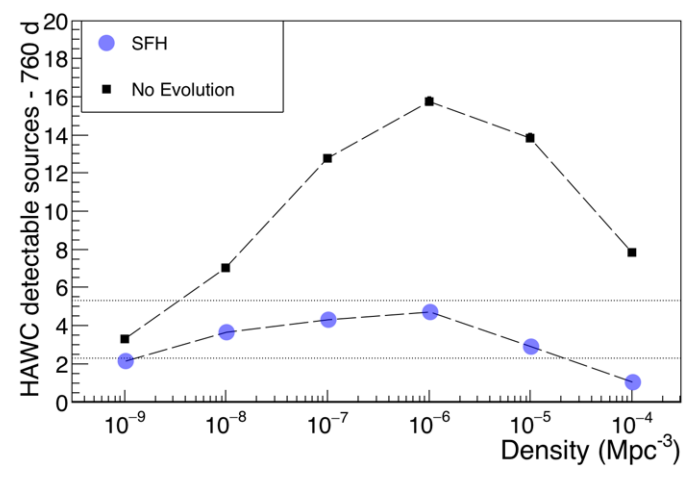

Figure 4: Predicted number of sources detectable by HAWC in 5 years of operation. Standard candles following SFH as well as no evolution.

\section{Discussion}

HAWC has observed two sources in the extragalactic sky. Are the results presented here consistent of not with that observation? The case of Mrk 421 and 501 being cosmic ray (and neutrino) sources is not settled. Both can be described with purely leptnic or with a mixed leptonic/hadronic model.

Let's assume that they are sources of neutrinos. Blazars, such as Mrk 421 and 501 do not have evolution in their density. As we found in $\S 6$, a wide range of densities is disfavored by HAWC for no evolution. Blazars, however, have evolution in their luminosity. We have not simulated this case in detail. Murase and Waxman, [17] define an "effective" local density for sources to take into account luminosity evolution. We will explore the case of blazars in more detail in future work.

Let's asume now that Mrk 421 and 501 are not neutrino sources. A broad range of densities, from $10^{-8}$ to $10^{-5} \mathrm{Mpc}^{-3}$, are disfavored, regardless of density evolution.

What characteristics the neutrino sources must have to avoid the constrains presented here: a) they can be transient - which we haven't studied yet b) the neutrino sources may be opaque to gamma rays c) there is a break in the neutrino spectrum at $\sim 10 \mathrm{TeV}$.

Our conclusions support previous work [9] that found that the astrophysical neutrino flux is too high in comparison with Fermi's extragalactic gamma ray background. Indeed Kistler [15] proposed the break at $10 \mathrm{TeV}$ (and matching $\mathrm{p}-\gamma$ neutrino production) to solve the incompatibility. At the highest densities, $\sim 10^{-5} \mathrm{Mpc}^{-3}$ HAWC is sensitive to gamma rays in the tens of $\mathrm{TeV}$ energy range, because sources are little or not affected by EBL. Hence at the highest densities, a break in the spectrum may not provide an answer to the apparent excess of neutrinos in comparison to gamma rays.

\section{Acknowledgements}

HAWC Collaboration: http://www.hawc-observatory.org/collaboration/icrc2017.php

\section{References}

[1] M. G. Aarsten et al. Evidence for high-energy extraterrestrial neutrinos at the icecube detector. Science, 342(6161), 2013. 
[2] M. G. Aarsten et al. A combined maximum-likelihood analysis of the high-energy astrophysical neutrino flux measured with icecube. The Astrophysical Journal, 809(1):98, 2015.

[3] M. G. Aartsen et al. Evidence for astrophysical muon neutrinos from the northern sky with icecube. Phys. Rev. Lett., 115:081102, Aug 2015.

[4] M. G. Aartsen et al. Search for prompt neutrino emission from gamma-ray bursts with icecube. The Astrophysical Journal Letters, 805(1):L5, 2015.

[5] M. G. Aartsen et al. All-sky search for time-integrated neutrino emission from astrophysical sources with 7 yr of icecube data. The Astrophysical Journal, 835(2):151, 2017.

[6] A. U. Abeysekara et al. The 2 hwc hawc observatory gamma-ray catalog. The Astrophysical Journal, 843(1):40, 2017.

[7] A. U. Abeysekara et al. Observation of the crab nebula with the hawc gamma-ray observatory. The Astrophysical Journal, 843(1):39, 2017.

[8] R. Atkins et al. Observation of tev gamma rays from the crab nebula with milagro using a new background rejection technique. The Astrophysical Journal, 595(2):803, 2003.

[9] K. Bechtol, M. Ahlers, M. D. Mauro, M. Ajello, and J. Vandenbroucke. Evidence against star-forming galaxies as the dominant source of icecube neutrinos. The Astrophysical Journal, 836(1):47, 2017.

[10] I. Collabooration. The icecube neutrino observatory - contributions to icrc 2015 part ii: Atmospheric and astrophysical diffuse neutrino searches of all flavors. In Proc. 34th International Cosmic Ray Conf., 2015.

[11] T. K. Gaisser. Cosmic Rays and Particle Physics. Cambridge University Press, 1 edition, 1990.

[12] R. C. Gilmore et al. Gev gamma-ray attenuation and the high-redshift uv background. Monthly Notices of the Royal Astronomical Society, 399(4):1694, 2009.

[13] T. Glüsenkamp et al. Analysis of the cumulative neutrino flux from fermi lat blazar populations using 3 years of icecube data. In Proc. Roma International Conference on Astroparticle Physics (RICAP-2014), volume 121, 2016.

[14] A. M. Hopkins and J. F. Beacom. On the normalization of the cosmic star formation history. The Astrophysical Journal, 651(1):142, 2006.

[15] M. D. Kistler. Problems and prospects from a flood of extragalactic tev neutrinos in icecube, 2015.

[16] M. Kowalski. Status of high-energy neutrino astronomy. Journal of Physics: Conference Series, 632(1):012039, 2015.

[17] K. Murase and E. Waxman. Constraining high-energy cosmic neutrino sources: Implications and prospects. Phys. Rev. D, 94:103006, Nov 2016.

[18] L. G. Strolger et al. The rate of core collapse supernovae to redshift 2.5 from the candels and clash supernova surveys. The Astrophysical Journal, 813(2):93, 2015.

[19] C. F. Tung and I. Taboada. Firesong. https://github.com/ChrisCFTung/FIRESONG, 2017. 